Note: This is a pre-copy-editing, author-produced PDF of an article accepted for publication in Journal of Intellectual Disability Research following peer review. The definitive publisher-authenticated version [Taggart L, McLaughlin D, Quinn B and Milligan V (2006), An exploration of substance misuse in people with intellectual disabilities, Journal of Intellectual Disability Research, 50(8), 588-597] is available online at

http://www3.interscience. wiley.com/journal/118574266/issue

\title{
An exploration of substance misuse in people with intellectual disabilities
}

\author{
L. Taggart, D. McLaughlin, B. Quinn \& V. Milligan \\ School of Nursing, University of Ulster, Northern Ireland, UK
}

Journal of Intellectual Disability Research 2006, 50(8), 588-597

Published by Blackwell Publishing Ltd

\begin{abstract}
Background: Little is known about the characteristics of people with intellectual disabilities (IDs) who misuse substances and how such problems impinge upon their well-being. The aim of this paper is to describe how alcohol and drugs affect the health of people with IDs.

Methods: A questionnaire was forwarded to all the community ID teams and mainstream addiction teams across Northern Ireland: 67 substance users with IDs and substantial substance-related problems were identified.

Results: Alcohol was found to be the main substance to be misused, with one-fifth of the substance users also found to be using a combination of illicit drugs and/or prescribed medication. Nearly three-quarters of the sample were found to be hazardously using alcohol for more than 5 years. Being male and young, having a borderline/mild ID, living independently and having a mental health problem were found to be risk factors for developing a 'substance related problem'. Various problematic behaviours were identified, including aggression, erratic mood changes, sexual exploitation, difficulties in maintaining relationships and loss of daily routine.

Conclusions: The findings of this study suggest that greater emphasis needs to be placed upon the early identification of this hidden population by primary and secondary healthcare personnel, and also ID personnel. Such early identification may also diminish the long-established patterns of use and associated related-behaviours that have been reported within this paper.
\end{abstract}

Keywords: identification, impact, intellectual disability, substance misuse

\section{Introduction}

The growth of 'substance misuse' in both the general and psychiatric populations appears to be mirrored in a growing trend for people with intellectual disabilities (IDs) who also hazardously use alcohol and drugs as identified by services (Degenhardt 2000; Sturmey et al 2003). However, there has been a dearth of literature that has comprehensively examined the 'use' and 'misuse' of these substances in people with IDs, and how this has impacted upon this population (Huxley et al 2005).

Despite the scant literature on this topic, 'substance use' in people with IDs is lower when compared with both the general and psychiatric populations (Annand \& Rus 1998; Burgard et al 2000; Havercamp \& Scandlin 2002; Sturmey et al 2003). Nevertheless, there remains variation in the reported prevalence rates in people with IDs who misuse alcohol and illicit drugs. Such discrepancies centre upon methodological problems concerning operational definitions, how such misuse is reported and whether the person is known to ID services or not (Clarke \& Wilson 1999; Sturmey et al 2003).

In a recent review of the literature pertaining to 'substance-related disorders' in this population, Sturmey et al (2003) stated that 'it is difficult to define any consensus among the studies as to the prevalence of alcohol misuse among people with intellectual disabilities, however, prevalence rates may vary somewhere between $0.5 \%$ and $2.0 \%$ of this population' (p.44). Figures for illicit drug misuse also indicate 
overall lower prevalence rates compared with alcohol abuse, with a prevalence rate of approximately one in 20 people with a borderline/mild ID (Gress \& Boss 1998; Pack et al 1998). This use of illicit drugs in people with IDs is also associated with where the person lives, in terms of both neighbourhood and with whom, age, local culture, ethnicity, availability and personal history (i.e. role models) (Sturmey et al 2003). Irrespective of the lack of consensus regarding prevalence, such substances have a detrimental impact on people with IDs, although previous studies have been mainly based on individual cases studies (Westermeyer et al 1988; Seminar Report by Poll 1998, Clarke \& Wilson 1999), with only a few studies utilizing staff reports on specific target populations (ARAC 2002; Wandsworth Drug \& Action Team 2003). Nonetheless, from these papers a number of specific health and behavioural issues have been correlated with hazardous substance misuse, including: physical health problems such as cardiovascular, respiratory tract and gastrointestinal problems (including sexual diseases and HIV); increased epileptic activity; a risk of violent behaviour and link with offending behaviour; more likely to have a co-morbid mental health problem; having higher levels of risk-taking behaviour (including suicide attempts); being exploited by others (including sexually) and greater likelihood of admission into a specialist hospital (Clarke \& Wilson 1999; Walkup et al 1999; Doody et al 2000; Mayer 2001; McGillivray \& Moore 2001; Stavrakaki 2002; Emerson \& Turnball 2005).

Given the difficulties in recognizing people with IDs who misuse a range of substances, this study aimed to identify all people known to services throughout Northern Ireland (population 1.8 million), one region of the UK. It involved all staff from both community ID and mainstream addiction teams across Northern Ireland. Two types of information were gathered: first, data on the numbers of people known to services; and second, information on the total population that give a more representative and detailed account of the characteristics of people with IDs who abused substances and the effects these substances had on them. This information will identify the types of services and supports required to meet the heterogeneous needs of this population; a population which has been both ignored and neglected by both ID and mainstream addiction services (ARAC 2002; Sturmey et al 2003).

\section{Methodology}

\section{Operational definition of substance abuse/misuse}

There are a number of substances that can be misused, which can have a broad range of effects on the people (i.e. physiological, psychological and social); these can also impinge upon their families and communities. This include alcohol, illicit drugs and overuse of prescribed medication, although other 'drugs' such as nicotine and caffeine can also be classified under the umbrella term of 'substances'. However, this study excluded these latter two substances from this investigation.

The definition of substance abuse in the DSM-4 Classification System (American Psychological Association 1994) was adapted in this study: 'the hazardous consumption of alcohol, illicit drugs and/or over use of prescribed medications which has been proven to be harmful to the persons' physical, psychological, interpersonal and social health within a 12-month period'. Aspects of the definition relating to role obligation, legal implications and hazardous tasks were deemed to be less relevant for people with IDs and crossed reference with Diagnostic Criterion for People with Learning Disabilities (DC-LD) (Royal College of Psychiatrists 2001).

\section{Informants}

Northern Ireland is divided into four Health and Social Service Boards; within these four Boards, there are 11 Community Health and Social Service Trusts. Each of these Trusts has six programmes of care: one is for people with IDs and another is for people with mental health problems of which addiction services are placed in. All the community ID and mainstream addiction team managers across these 11 community Trusts were forwarded a letter explaining the nature of the project. Enclosed were 10 questionnaires for these managers to distribute to the community personnel on their team who had a person(s) with an ID that was abusing substances on their caseload.

In total, 54 community informants [i.e. social workers $(n=31)$ and nurses $(n=18)$ and mainstream addiction workers $(n=4)$ ] self-completed the questionnaire. There were 42 female $(77.7 \%)$ and 12 male (22.3\%) informants: several informants completed a questionnaire on more than one person. The response rate was found to be varied across these Trusts from both the ID and addiction teams. A 
number of staff from the nine community Trusts offering services to people with IDs out of the 11 Trusts responded. The numbers of substance users varied within each of these nine Trusts, ranging from 14 substance users in one inner-city area to three people in one rural area. Of the eight community addiction teams that exist across Northern Ireland, staff responded from only three of these teams. There were two informants working within one inner city area with a person with an ID and addiction problems, and two other staff into two other teams who also reported working with similar substance users. However, five of these addiction teams did not respond.

As a result of no questionnaires being returned from the other five addiction teams and the two community Trusts for people with IDs, the managers of these teams were further contacted. They informed the researchers that they currently had no individuals that they were aware of on their and on their staff's caseloads who had an ID and substance-related issues. In spite of the bias from the variance of the response rates, these findings demonstrate that a person with an ID and a substance-related problem(s) is more likely to receive treatment/services from ID services as opposed to the specialist addiction services.

\section{Materials}

Initial meetings took place with two community ID teams to discuss some of the issues pertaining to services users who were misusing substances. On the basis of these two informal discussions and a review of the literature, a questionnaire was drafted. A self-completed questionnaire was developed so that information could be obtained on a larger sample of substance users from across Northern Ireland. This was divided into three parts. The first section sought information regarding the community informant who completed the questionnaire. The second section asked questions about the people misusing the substances (i.e. age, gender, where they live, level of ID and any health problems). Third, information was sought on the type of substances these people were misusing (i.e. length of use, how the substance affected their health, including relationships with family, peers and professional staff, and daily routine). The questionnaire was then piloted with five informants and minor changes made.

\section{Procedure}

An information letter about the project was sent to all the managers of the community ID teams and addiction teams along with the questionnaires for distribution. Each informant was reminded to ensure that no identifiable information was written on the questionnaire. Furthermore, to diminish the possibility of duplicating (i.e. two questionnaires being completed by a person's social worker and community nurse), informants were asked to check with their colleagues prior to the completion of a questionnaire. Similarly, both groups of informants were asked to check with their colleagues either in the addiction services or in the ID services, whether the other service was also working with the person, again to avoid duplication.

\section{Ethical considerations}

The Health and Personal Social Services Research Ethics Committee in Northern Ireland granted ethical approval for this project in terms of collating and transferring confidential information anonymously on individuals from different community personnel to the research team. The information was coded within SPSS and Word files. These computer files contained no identifiable information about the participants and paper records were destroyed.

\section{Results}

\section{Substance users}

The community informants reported upon a total of 67 adults with IDs who were identified to be misusing a range of substances resulting in substantial problems: 41 men (61.2\%) and 26 women (38.8\%). Based upon the informants' completion of the questionnaires and compared with the prevalence rate of ID for adults across Northern Ireland, this figure can be equated to $0.8 \%$ of the adult ID population who were known to services (McConkey et al. 2006); however, this may not reflect an accurate prevalence rate.

This study found that 18 users (26.9\%) were aged 50 years plus, $12(17.9 \%)$ aged $41-50$ years, another $18(26.9 \%)$ aged $31-40$ years, $16(23.9 \%)$ aged $21-30$ years, and 3 users $(4.5 \%)$ were aged $18-20$ years. Overall, 15 male users (36.6\%) were below the age of 30 years compared with four female 
(15.4\%). Based upon the informants' knowledge of the individual on their caseload, they identified 11 users (16.4\%) as having a borderline ID, $39(58.2 \%)$ as having a mild ID and $17(25.6 \%)$ as having a moderate ID, using ICD-10 criteria. No users were identified to have a severe/profound ID. No differences were found between the level of ID across gender and age. However, these results reflect a different profile of service users compared with the wider population of people with IDs, particularly as McConkey et al. (2006) found that $70 \%$ of people known to services had a moderate/severe/profound disability and only $30 \%$ had a mild/borderline ID.

Just less than half of the substance users $[n=30(44.8 \%)]$ lived independently within their own home, 5 $(7.4 \%)$ of whom were living with their partner. For the remaining participants, 20 users $(29.9 \%)$ lived with their family, 15 users (22.4\%) resided within a supported living scheme and 2 users (3\%) lived within a residential facility. These findings highlight a more independent population in comparison with the reported living arrangements for people with IDs across Ireland, Northern Ireland and the rest of the UK, in which $9 \%$ of people were known to ID services live independently (Emerson et al. 1999; McConkey et al. 2006).

\section{Associated health problems}

The informants were asked to comment on the users' health. The main additional problem identified was that of mental health, with 18 users $(26.9 \%)$ reported to have a diagnosed psychiatric disorder and a further $8(11.9 \%)$ suspected to have a mental health problem. The most common was that of a psychotic disorder followed by an affective disorder. Alongside this, one participant had a diagnosis of 'Alcohol Dependence Syndrome'; this can also be considered a co-morbid psychiatric condition. No significant differences were found regarding age, gender and level of disability with mental health status.

A variety of physical health problems were also identified for 16 users (23.9\%), including angina, asthma, diabetes, asthma, gout, arthritis, high blood pressure and an unsteady gait. A sensory disability was noted for 7 users (10.4\%), and 6 users (9\%) were reported to have epilepsy. Overall, this account highlights a sub-population of people with IDs that are not reflective of normal demographic profile of people with IDs that have been reported previously.

\section{Information on substance misuse}

Alcohol was reported to be misused by all of the users (100\%). Of the 67 users, $13(19.4 \%)$ were also reported to be misusing other substances such as illicit drugs and/or overusing prescribed medications, including cannabis $(n=8)$, prescribed medications [i.e. paracetamol, lbruofen, diazepam, Kepak (i.e. painkiller)] $(n=4)$, Ecstasy $(n=3)$, amphetamines $(n=1)$, solvents $(n=1)$ and cocaine $(n=1)$. One participant was also described to be addicted to 'gambling machines'.

The informants reported that for those 13 users misusing both alcohol and a combination of illicit drugs and/or prescribed medications, they were significantly more likely to be 30 years and below $[n=10$ (76.9\%) $\left.\left(\mathrm{X}^{2}=18.7, P<0.001\right)\right]$; to be male $\left[n=11(84.6 \%), \mathrm{X}^{2}=3.9, P<0.05\right]$ and to live independently [ $\left.n=9(69.2 \%), x^{2}=3.9, P<0.05\right]$. No differences were found between the two groups relating to their level of ID and mental health status.

The frequency of the alcohol and drug misuse was as follows: 21 users (31.3\%) misused on a daily basis; 11 users (16.4\%) misused at weekends; 9 users (13.4\%) misused approximately every 3-4 days; and 13 users (19.4\%) misused on a weekly pattern. For 13 users (19.4\%), the informants were not able to provide clear information on the patterns of misuse, as all of these individuals lived in their own home independently.

In all, $73.1 \%$ of the users $(n=49)$ were reported to be misusing substances for over 5 years; this was followed by $22.4 \%(n=15)$ misusing such substances between 2 and 5 years. Three users $(4.5 \%)$ were cited to be misusing substances between 1 and 2 years. Female users [ $n=22(84.6 \%)]$, compared with male users [ $n=27(65.9 \%)]$, were more likely to be abusing alcohol for over 5 years, although this was not statistically significant. No differences were found regarding length of use across age, level of disability and place of abode. The length of abuse (over 5 years) was significantly greater for those users who misused alcohol only $[n=42(77.8 \%)]$, compared with those reported to abuse both alcohol and drugs $[n=7(53.8 \%)](F=5.04$, d.f. $=1, P<0.05)$. 


\section{Effects of the misuse on the substance users' behaviour}

Table 1 shows a wide range of behaviours that the informants reported occurred when the users were under the influence of the substances identified above. 'Verbal aggression' (i.e. being 'demanding' 'argumentative' and 'confrontational') was reported for over two-thirds (70.1\%) of service users as were 'mood changes' (ranged from 'feelings of worthlessness', 'despair', feeling 'depressed', having 'low motivation', 'poor social judgements' to 'less cooperative'). Those users identified as having a psychiatric disorder, and also suspected of having a mental health problem (44.7\%), were more likely to have unpredictable 'mood changes' compared with those not reported to have a mental health problem $(11.1 \%)\left(X^{2}=3.7, P<0.05\right)$.

Just less than half of the users were reportedly 'exploited by others' (46.3\%), and this was significantly more so with women $(82.6 \%)$ compared with men $(46.2 \%)\left(x^{2}=6.9, P<0.01\right)$. These included sexual, physical, psychological and financial exploitation. Furthermore, three female users were reported to have made 'allegations of sexual harassment against unknown males' while under the influence of substances.

'Physical aggression' to formal or informal carers, other people with IDs and members of the public, were identified as problematic for nearly half of the users. Over one-third of the substance users would frequently attend the local A \& E departments while under the influence of the alcohol; this was significantly more so for those substance users who were also reported to 'physically injure-self ' (19.4\%) $\left(X^{2}=8.3, P<0.01\right)$.

Moreover, just less than a third of the substance users reported having 'suicidal ideations/thoughts' when drinking (see Table 1). Substance users identified as having a psychiatric disorder, and also suspected of having a mental health problem (63.2\%), were more likely to have 'suicidal thoughts' compared with those not reported to have a mental health problem $(33.3 \%)\left(x^{2}=3.9, P<0.05\right)$.

Around one-third of the substance users were reported to get into trouble with the police/law (i.e. for 'antisocial behaviours', 'shop lifting') (see Table 1). Male users (56.7\%) were more likely to get caught up in 'offending behaviour' compared with female users $(30 \%)\left(X^{2}=3.4, P<0.05\right)$. One participant was reported to get involved in 'paramilitary activities' often associated with 'periods of heavy drinking'.

Table 1 The effects of the misuse on the substance users

\begin{tabular}{lcc}
\hline Behaviour & $\begin{array}{c}\text { No. of cases } \\
(\boldsymbol{n}=\mathbf{6 7})\end{array}$ & $\%$ \\
\hline Verbal aggression & 47 & 70.1 \\
Changes in mood & 47 & 70.1 \\
Exploited by others & 31 & 46.3 \\
Physical aggression & 30 & 44.8 \\
Attending A \& E department & 24 & 35.8 \\
Offending behaviour & 23 & 34.3 \\
Suicidal ideations/thoughts & 19 & 28.4 \\
Rows with care staff & 16 & 23.9 \\
Physically injures self & 13 & 19.4 \\
Rows with partner/family member & 13 & 19.4 \\
Overdosing on prescribed medication & 11 & 16.4 \\
Other & 9 & 13.3 \\
Exploiting others & 7 & 10.4 \\
Increases in epileptic activity & 4 & 6.0 \\
\hline
\end{tabular}

Comments from the informants emphasized the significance of the effects of the substance abuse upon the users' physical and psychological health:

$\mathrm{N}$ has been admitted to hospital due to an attempted overdose of painkillers due to excessive drinking which has been brought on by erratic mood swings.

$\mathrm{N}$ has regular thoughts of self-harm after drinking; he can also become non-compliant with his medication at times.

When binge drinking, $\mathrm{N}$ neglects all aspects of his health becoming non-compliant with prescribed medication, becomes doubly incontinent, has low self-esteem and has thoughts of suicide. 
The median number of problematic behaviours reported was 4 , with a minimum of 1 and a maximum of 9 . However, no differences were observed regarding the median number of problematic behaviours across gender, level of ID and place of abode. Similarly, no differences were found between those substance users who abused alcohol only and those who misused both alcohol and drugs. Nonetheless, these figures present a distressing and worrying account of a population with multiple complex needs.

\section{Effects of substance misuse on the users' relationships}

The community informants reported numerous difficulties in their relationships with carers and peers that arose from the people's behaviours as a direct consequence of the hazardous misuse. These mainly constituted 'verbal aggression', 'threatening behaviour' and often 'getting into rows'. Problems also were reported regarding maintaining positive relationships with their partners, parents and siblings, and professional carers. The community informants also reported problems in engaging the participant in health education/promotion programmes because of these difficulties.

With regards to peers, the informants reported marked difficulties for the users in maintaining friendships. When the users had friends, these may not be positive relationships, rather they are characterized by unequal relationships based upon financial and sexual exploitation. Below are some of the informants' statements:

$\mathrm{N}$ is exploited by those he drinks with, they abuse his hospitality by spending long periods of time in his home taking advantage of him financially.

$\mathrm{N}$ has lost all his friends, he is not welcome in the pubs and clubs and has been the victim of taunting, stoning and beatings around certain areas of the city while drinking.

N's friends are transient alcohol and drug misusers who financially exploit this woman regularly.

\section{Effects of substance misuse on the users' daily routine}

Only 12 users (17.9\%) were reported to engage in some form of daily routine such as supported employment or attending a day centre mainly on a part-time basis. The remaining 55 users $(82.1 \%)$ did not avail of any form of formal structured activities. The reasons given ranged from 'continual drinking patterns', 'refusal to get out of bed', 'reluctance to attend', 'poor co-operation', 'low motivation' to the work placement being 'suspended'. 'Isolation' and 'boredom' often resulted, leading some users to spend large parts of their day on their own drinking. Some comments from the informants included:

The client is socially isolated, occasionally goes to a friend's house that lives two doors away to drink. $\mathrm{N}$ refuses to attend any day centres spending the day alone in room watching television or listening to music.

$\mathrm{N}$ tends to go out to buy beer then lie upstairs in bed drinking this. $\mathrm{N}$ does not wash and does not attend day care. Spends time at home watching and viewing from window and sits in the kitchen.

\section{Discussion}

This study found a varying response rate from the community informants in both the ID and the mainstream addiction teams, and also across geographic regions in Northern Ireland. Whether this was a result of few people using these services or whether staff within the mainstream addiction settings are unsure if the person had a diagnosed ID, and significant problems with alcohol or other substances, is unclear.

Alongside this obscurity that some staff had in recognizing this population, three-quarters of the users who were known to services have been hazardously using substances for more than 5 years, frequently associated with a range of distressing negative behaviours as reported in Table 1 . These findings highlight the secretiveness and resilience of the users' behaviours to avoid detection particularly as many were found live independently. Another difficulty that service personnel may regularly encounter is the issue of the service user being in 'denial' of having a substance-related problem and therefore not proactively seeking help. Family carers may also be reluctant to report the service users' behaviours in case they are hospitalized and/or lose their independence. This concealed and enduring use within this population highlights the importance for the early recognition of these problems in order that an accurate diagnosis can be made and appropriate treatment packages developed. Future studies may also need to 
include people who do not use ID and mainstream addiction services, in an attempt to be fully inclusive thereby providing data on more accurate prevalence rates.

How this 'hidden population' is identified will require general practitioners and others in the primary and secondary care settings, and also ID personnel (i.e. teachers and staff in residential/day care/ employment facilities), to recognize those people with IDs who are hazardously using substances earlier than has been identified within this study. Yet, these key professionals in primary care settings such as general practitioners have been shown to be lacking in knowledge and skills to identify, and also to offer effective care to, people with substance issues (Martin 1996; McGillion et al. 2000; Mistral \& Velleman 2001): a situation that is further compounded when the person also has an ID.

This study identified 67 people with IDs who had a substance-related problem; an estimated $0.8 \%$ of the adult population with IDs (McConkey et al. 2006). This figure may be a lower estimate than the true prevalence rate, as the figure of $0.8 \%$ is only based upon those informants who completed a questionnaire and those substance users who are known to such services. Of those people that are not in contact with intellectual services, they are more likely to have a borderline/mild ID: a subgroup of people who may be at a greater risk from developing a substance-related problem (Christian \& Poling 1997; Robertson et al. 2000; Sturmey et al. 2003).

In addition, this figure of $0.8 \%$ could be inflated, given the impact that the 'troubles' has had on the nondisabled population within Northern Ireland and how this population has used substances to cope; yet, no systematic studies have examined the impact of the 'troubles' on people with IDs. This study further found a higher proportion of people with IDs to use a range of substances in one inner-city area compared with rural areas. Whether this was the cause of a 35-year history of the 'troubles' is difficult to assert, particularly given the long term and striking high levels of socio-economic deprivation also found within this specific area.

The majority of the users known to services had a borderline/mild ID, were male and tended to be younger, with the majority hazardously using alcohol for more than 5 years. Alongside a diagnosis of an ID, $26.9 \%$ of the users were reported to have a psychiatric disorder with another $11.9 \%$ suspected to have a mental health problem. These findings support previous studies that examined this 'triple diagnosis' as described by Barnhill (2000). Although people with IDs are more likely to have a mental health problem compared with the general population, whether this exacerbates the people's substancerelated patterns, and behavioural problems, remains unclear and requires further scrutiny. Nonetheless, these results strongly emphasize the importance for early screening for mental health problems and alcohol/drugs in this population, particularly as this 'triple diagnosis' can lead to further complications in providing an effective treatment package. This may help to identify the causality of the relationship that coexists between these conditions.

Over two-thirds of the users were living either fully or partially independently in the community, thus independent community living may be an important risk factor in identifying those individuals who go on to develop long-term, substance-related problems.

'Alcohol' was the main substance abused by all the users, with a fifth also using illicit drugs and prescribed medications. Moreover, all of these 67 users, according to the informants, have met criterion taken from DSM-4 (American Psychological Association 1994), including symptoms that have been observed for more than 12 months. However, only one user had received a diagnosis of an 'Alcohol Dependence Syndrome'. These results demonstrate how the majority of people within this study have not been identified to have a diagnosed 'substance related dependence problem', yet have been abusing substances for over 5 years with considerable negative consequences as reported in Table 1; this finding highlights the continued unmet need of this population.

The findings above have clearly demonstrated the distressing pattern of the broad range of substancerelated problematic behaviours that have profoundly impacted upon the people's well-being and that of their carers. This study also found that the majority of the users had interpersonal difficulties. More alarmingly, over half of the service users were reported to have been 'exploited' by their peers: mainly non-disabled peers (i.e. physically, psychologically, 'financially' and also 'sexually'). Alongside this, the majority of the users were unable to sustain their daily activities, with many left feeling 'isolated' and 
'bored'. This excessive amount of unstructured free time can also predispose, precipitate and maintain this population to misuse substances to further fill this void. Service providers will need to re-examine the current services that they provide and the treatment packages that are offered to this population in light of these multifaceted issues raised in this study.

These results stress the complexities that both ID and mainstream addiction staff face in promoting positive health, recognizing, assessing, treating and managing this population within a community setting. Community personnel will require the appropriate supports, resources and training to be in place so that they can quickly and promptly identify this population, thereby offering an effective treatment package.

But debate continues with regards to which service is best placed to serve this population? Despite ID personnel having the competence and confidence in addressing the generic needs of this population, however, these personnel overall lack the specialized skills to manage the people's substance-related issues. Similarly, mainstream addiction staff have the competence to manage those people with substance-related problems; yet lack the skills to work with people with IDs who also misuse substances. It is also worth noting that for the majority of the service users within this study, mainstream addiction personnel were not involved in the co-working and management of this population with their ID colleagues. Moreover, no clear frameworks on how to manage this population from policy planners and service providers with little, if any, interagency collaboration between ID and mainstream addiction service providers are available across the UK (ARAC 2002; Wandsworth Drug \& Action Team 2003, Huxley et al. 2003). Nevertheless, this study indicates that as the majority of people with IDs who misuse substances have a borderline/mild ID and successfully live independently, many of this population could therefore access mainstream addiction services if given the opportunity.

Certain shortcomings should be acknowledged within this study. First, this study reported upon a sample size of 67 substance users; this is not to say that all users have been represented within this sample, as only those known to services have been included. Second, this study, although being one of the largest studies undertaken within this area, is constrained, as not all the community Trusts for people with IDs responded. Similarly, only three out of the eight addiction teams also forwarded questionnaires; therefore, these results should be tentatively interpreted as the prevalence rate may be an underestimate of the true prevalence rate of substance-related problems in this population. Third, this study examined the reports of community informants regarding the effects that substances has upon the people's wellbeing; it therefore failed to incorporate the views of the substance user. Undertaking such an in-depth study in the future will require researchers to gain access to a number of people with IDs who misuse substances and more importantly who are known to services: a population that can be difficult to identify and a population who may not be willing to engage (Rivinius 1988).

\section{Conclusion}

This study has shown that substance misuse exists in people with mostly borderline and mild IDs, and that such misuse has significant health and social consequences for this population; including those consequences for their carers. This co-morbidity is associated with mental health problems and requires a re-examination of existing services: especially when more people with IDs are being supported to live in the community and encouraged to utilize mainstream services. A strong focus needs to be placed upon primary care personnel and ID staff to recognize those individuals who are hazardously using alcohol, drugs and/or prescribed medications. Such early identification may also diminish the long-established patterns of use and associated behaviours that have been described within this paper.

\section{Acknowledgements}

This research was funded by a research grant awarded by the Mental Health and Learning Disability Review (Northern Ireland). We are grateful to the all the community informants that took part in this study. We are also thankful to Clare McFarlane who was a psychology student on placement with the first author and assisted with the project. 


\section{References}

Addictions Resource Agency for Commissioners (ARAC) (2002) Substance Misuse and People with Learning Disabilities. Report commissioned by Merton Drug and Alcohol Action Team.

American Psychological Association (1994) Diagnostic Statistical Manual of Mental Disorders (4th edition). DSM-4. American Psychiatric Association, Washington, DC.

Annand G. N. \& Rus G. (1998) Over-coming barriers to effective treatment for persons with mental retardation and substance related problems. NADD Bulletin 1, 14-17 $\square$.

Barnhill J. (2000) Triple diagnosis: substance abuse, mental illness and mental retardation. In: Proceedings of the NADD International Conference: New York.

Burgard J. F., Donohue B., Azrin N. A. \& Teichner G. (2000) Prevalence and treatment of substance abuse in the mentally retarded population: an empirical review. Journal of Psychoactive Drugs 32, 293-8.

Christian L. \& Poling A. (1997) Drug abuse in persons with mental retardation: a review. American Journal on Mental Retardation 102, 126-36.

Clarke J. J. \& Wilson D. N. (1999) Alcohol problems and intellectual disability. Journal of Intellectual Disability Research 43, 135-9.

Degenhardt L. (2000) Interventions for people with alcohol use disorders and an intellectual disability: a review of the literature. Journal of Intellectual and Developmental Disability 25, 133-46.

Doody G. A., Thompson L. D., Miller P. \& Johnstone E. C. (2000) Predictors of admissions to a high security hospital for people with intellectual disabilities. Journal of Intellectual Disability Research 44, 130-7.

Emerson E., Robertson J., Gregory N., Hatton C., Kessissoglou S., Hallam A., Knapp M., Järbrink K., Netten A. \& Walsh P. (1999) A Comparative Analysis of Quality and Costs in Village Communities, Residential Campuses and Dispersed Housing Schemes. Hester Adrian Centre, Manchester.

Emerson E. \& Turnball L. (2005) Self-reported smoking and alcohol use among adolescents with a mild intellectual disability. Journal of Intellectual Disabilities 9, 58-69.

Gress J. R. \& Boss M. S. (1996) Substance abuse differences among students receiving special education school services. Child Psychiatry and Human Development 26, 235-46.

Havercamp S. \& Scandlin D. (2002) Sex and Sadness: Surveillance of Mood Disorders as a Function of Gender. Paper Presented to the Annual Conference of the National Association For Dual Diagnosis, Denver, Co.

Huxley A., Copello A. \& Day E. (2005) Substance misuse and the need for integrated services. Learning Disability Practice 6, 14-17.

McConkey R., Mulvany F. \& Barron S. (2006) Adult persons with intellectual disability on the island of Ireland. Journal of Intellectual Disability (in press).

McGillion J., Wanigaratne S., Feinmann C., Godden T. \& Byren A. (2000) GPs' attitudes towards the treatment of drug misusers. British Journal of General Practice 50, 385-6.

McGillivray J. A. \& Moore M. R. (2001) Substance use by offenders with mild intellectual disability. Journal of Intellectual and Developmental Disabilities 26, 197-210.

Martin E. (1996) Training in substance abuse is lacking for GP's. British Medical Journal 312, 186-7.

Mayer M. A. (2001) SAMIRIS: substance abusers who have both mental illness and mental retardation. NADD Bulletin 4, 92-9.

Mistral W. \& Velleman R. (2001) Substance-misusing patients in primary care: incidences, services provided and problems. A survey of general practitioners in Wiltshire. Drugs: Education, Prevention and Policy 8, 61-72.

Pack R. P., Wallander J. L. \& Browne D. (1998) Health risks behaviours of African-American adolescents with mild mental retardation. American Journal on Mental Retardation 102, 409-420.

Rivinius T. (1988) Alcohol use disorder in mentally retarded persons. Psychiatric Aspects of Mental Retardation Reviews 7, 19-26.

Robertson J., Emerson E., Gregory N., Hatton C., Turner S., Kessissoglou S. \& Hallam A. (2000) Lifestyle related risk factors for poor health in residential settings for people with intellectual disabilities. Research in Developmental Disabilities 21, 469-86.

Royal College of Psychiatrists (2001) DC-LD: Diagnostic Criteria for Psychiatric Disorders for Use with Adults with Learning Disabilities/Mental Retardation. Gaskell, London.

Seminar Report by Poll (1998) Coming for a Drink? A report of a Seminar on Alcohol Misuse and People with Learning Difficulties. The Elfrida Society, London. 
Stavrakaki C. (2002) Substance-related disorders in persons with a developmental disability. In: Dual Diagnosis: An Introduction to the Mental Health Needs of Persons with Developmental Disabilities (eds D. M. Griffiths, C. Stavrakaki \& J. Summers), pp. 455-509. NADD, New York.

Sturmey P., Reyer H., Lee R. \& Robek A. (2003) Substance Related Disorders in Persons with Mental Retardation. NADD, Kingston, NY.

Walkup J., Sambamoorthi U. \& Crystal S. (1999) Characteristics of persons with mental retardation with HIV/ AIDS infection in a statewide Medicaid population. American Journal on Mental Retardation 104, 356-63.

Wandsworth Drug \& Action Team (2003) Drug and alcohol abuse by adults with learning disability in the Borough of Wandsworth. Undertaken by NASH.

Westermeyer J., Phaobtang T. \& Neider J. (1988) Substance use and abuse among mentally retarded persons: a comparison of patients and a survey population. American Journal of Drug and Alcohol Abuse 14, 109-23. 